\title{
Solving TSP Based On the Improved Simulated Annealing Algorithm with Sequential Access Restrictions
}

\author{
Lin Xiong ${ }^{1,2, a^{*}}$ and Shunxin $\mathrm{Li}^{1,2, \mathrm{~b}}$ \\ ${ }^{1}$ College of Computer Science \& Technology, Wuhan University of Science \& Technology, China \\ ${ }^{2}$ Hubei Province Key Laboratory of Intelligent Information Processing and Real-time Industrial \\ System, China \\ a977645341@qq.com, blishunxin72@163.com
}

Keywords: TSP problem; Combinatorial optimization; Sequential access restrictions; Clustering; Simulated annealing

\begin{abstract}
TSP problem is a typical combinatorial optimization problem, which has been applied in many fields in real life. In the solution of TSP, it is no doubt that it will reduce some unnecessary calculations if the approximate path is determined in advance. Sequential access restrictions combined with the properties of convex hull, gives roughly access order for TSP. Applying access restrictions to the path planning algorithm of TSP, clustering analysis the initial points with access restrictions, combing the clustering results with simulated annealing algorithm, and improving the transform rules of solutions in simulated annealing, an improved simulated annealing algorithm is proposed based on access restrictions was proposed. The proposed algorithm shortens the operation time, improves the convergence efficiency of the simulated annealing algorithm, and obtains a good experimental result.
\end{abstract}

\section{Introduction}

Traveling Salesman Problem (TSP) [1] can be described as: find the shortest closed loop of a city node set, each city can only visit one time, and eventually return to the initial city node. A lot of problems in real life can be simplified as the traveling salesman problem, because it is widely used in the fields of computer application, operations research and engineering optimization, therefore, it has attracted a lot of researchers in many fields for a long time to use a variety of means to solve and optimize it.

Simulated annealing algorithm (SA)[2] as a typical intelligent optimization algorithm, by giving the search process a time variant and ultimately tends to zero probability of sudden jump, thus effectively avoid falling into local minimum and finally tends to the global optimal. Although the SA algorithm converges to the global optimal solution in theory, but its convergence rate is slow. Currently, the simulated annealing algorithm has been widely used in many fields, such as VLSI, production scheduling, control engineering, machine learning, neural networks and signal processing.

In view of the characteristics of global optimization, SA algorithm is widely used in the research of TSP problem. The SA algorithm is improved by the method of reverse operation, which expands the search scope and improves the ability of global optimization in literature [3]. Memory function is added in the literature [4], which can reduce the calculation time effectively. In addition, the tabu list is added to the literature [5], which reduces the possibility of repeated computation and improves the convergence efficiency. And in the literature [6-10], there are some intelligent optimization algorithms are combined with SA algorithm, although the global optimization ability of SA algorithm is highlighted., but the convergence and running time of the algorithm still waits for further enhancing.

Cluster analysis [11] aims to divide a large data set into several groups according to the similarity of the data, for insight into the correlation of it. The clustering analysis which has been widely used in business intelligence, pattern recognition, image web search, biology, security and other areas, has very high research significance for the discovery of the potential relationship between data. 
A more difficult problem is to select what kind of operator to transform the solution, not only to ensure the global convergence of the algorithm, but also can improve the ability of local search and reduce the unnecessary calculation process of the SA algorithm. In this paper, we propose a new hybrid clustering simulated annealing algorithm. Which taking into account the characteristics of the nodes in the Euclidean plane, combined with the sequential access restrictions ${ }^{[12]}$, and mix with the idea of clustering classification. At last, we compare and analyze the different algorithms.

\section{Access Sequential Restriction}

Access sequential restriction theorem proposed by Flood ${ }^{[12]}$, by using the convex hull it proved that the necessary condition for solving the TSP's shortest loop. The description of this theorem is as follows.

$\mathrm{V}$ is the set of a given set of cities, $\mathrm{T}$ is the convex hull of $\mathrm{V}, \mathrm{L}$ is the shortest loop of $\mathrm{V}$. Sequential access to city nodes in $\mathrm{T}$ according to the order of $\mathrm{L}$, so get a polygon $\mathrm{F}$. Then polygon $\mathrm{F}$ and convex hull $\mathrm{T}$ are equivalent.

As shown in Fig. 1, the solid line shows the shortest loop in the Euclidean plane of 12 points, the dotted line shows the convex hull composed of by a series of points. It can be seen form the figure, the sequence of nodes on the dotted line and solid line loop is the same.

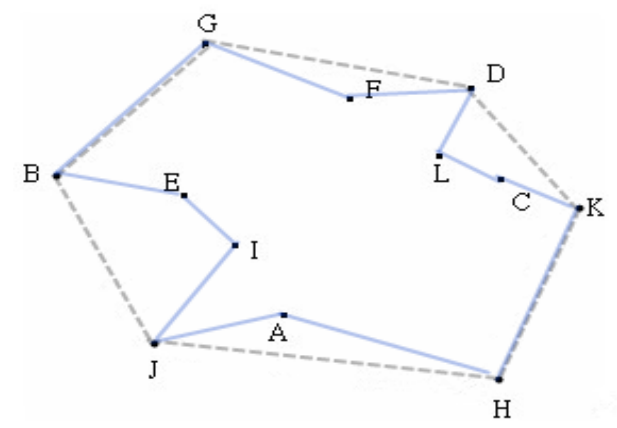

Figure 1. The shortest circuit and convex hull

The following inference can be derived from the access sequential restriction theorem:

In the shortest Hamiltonian circuit, part of the nodes' sequence is known, namely circle around the convex hull.

To find the shortest loop is equivalent to add some other city node on each side of the convex hull.

\section{Cluster Analysis}

Distance Based Clustering Analysis. Distance based clustering analysis is to determine the similarity between the two objects with the distance between them, and the distance can be defined in Euclidean space, vector space, road network or other space. The main clustering methods include partitioning method, hierarchical method, density based method and grid based method.

As shown in Fig. 2, we show that several data points in the Euclidean space are clustered according to the distribution.

Clustering analysis based on distance can better find all data points of the nearest neighbor domain. The nodes in the rectangular box (shown in Fig. 2) are bound to be closely connected, and they are bound to be closely connected to the whole loop of the TSP problem. That is to say: because the density of centralized data within a cluster contains, in the solution of the TSP problem, we can consider the cluster as a whole, and ignore its internal permutations and combinations. 


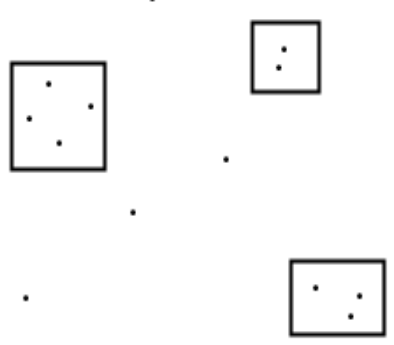

Figure 2. Cluster analysis in Euclidean plane

Measure of Distance. The distance of the data point $\mathrm{p}$ to the cluster $\mathrm{C}$ is measured with maximum distance, and the formula is as follows:

$$
\operatorname{dis}(p, C)=\max _{p^{\prime} \in C}\left\{\left|p-p^{\prime}\right|\right\}
$$

Division for the next data point $\mathrm{p}$, we use the minimum partition method, namely:

$$
D_{\text {min }}=\min _{C \in U, C^{\prime} \in U}\left\{\operatorname{dis}(p, C), \operatorname{dis}\left(p, C^{\prime}\right)\right\}
$$

According to the above formula, we can obtain the closest cluster $\mathrm{C}$ to the data point $\mathrm{p}$, and add point $\mathrm{p}$ to cluster $\mathrm{C}$.

The above method obtained data point in the same cluster is concentration of density, in terms of the traditional method like k- mean and k- center method, without having to recalculate the cluster centers.

Cluster Initialization. Each data point in the convex hull is a series of initial cluster as above sequential access restrictions referred to.

The benefit of classified according to the convex hull is: in the solution to TSP, the order of these clusters in accordance with the sequential access restriction, therefore only need to compute the different permutation and combination of different clusters' internal data points, without having to consider the order of these clusters.

The following are the classification results of data sets of TSP Lib oliver30 (as shown in Fig. 3).

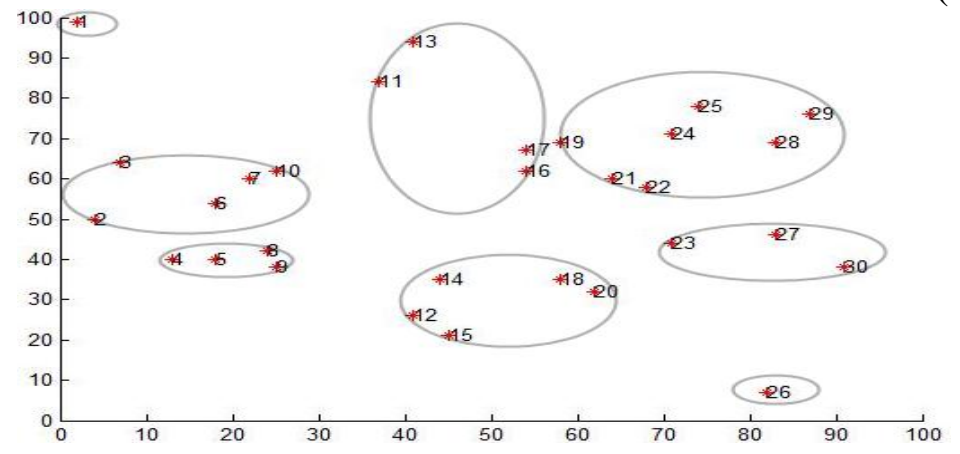

Figure 3. TSP Lib oliver30 division results

\section{SA with Sequential Access Restrictions}

Improved SA Algorithm. The simulated annealing method starting from a certain initial solution, and after a large number of transformations, the relative optimal solutions of the optimal control parameters can be obtained. Repeat Metropolis process, finally able to get the global optimal solution.

SA algorithm depends on iterative approach of the neighborhood structure, and how to find a neighborhood solution directly affect the convergence speed and global optimal solution. This means that the transformation of the solution is very important to solve the problem. In the process of the transformation of the traditional simulated annealing algorithm solution with the method of 
exchange or reverse.

Only by exchanging the positions of the two nodes, it's simple, but easy to fall into local optimal solution; and reverse all the nodes between the two nodes while it is possible to avoid falling into local optimal solution, but the convergence speed is slow. Aim at this problem, this paper proposes a new method to solve the problem, which is based on the reverse method, combined with the idea of access sequence and clustering, and finally puts forward a new method to generate the initial solution and convert it.

Generation of Initial Solution. Clustering makes all the nodes in the same cluster have similar properties. For each node in the Euclidean plane of dividing and hypothesis node distances between each block relative to the nodes within a block of the distance is large, then the nodes of the shortest circuit can regarded as each block according to a certain order of arrangement.

In this paper, combined with the sequential access restriction theorem and the idea of clustering, the generation of the initial solution is improved.

Taking the oliver30 data set in tsp lib as an example, this data set contains 30 points,

The data set contains 30 points, respectively represented by the serial number of 1-30, the sequence of the convex hull is $\{26,15,4,2,1,13,29,30\}$. Let the point in this sequence as the starting point and the above 30 points are divided into clusters according to the above clustering method, the results are: $23\}$.

$\{26\},\{15,12,14,18,20\},\{4,5,8,9\},\{2,3,6,7,10\},\{1\},\{13,11,17,16\},\{29,28,25,24,22,21,19\},\{30,27$,

Then, the data points in each cluster are randomly arranged, the order of the clusters is constant, and the initial solution is obtained.

Transformation of the Solution. In this paper, the transformation of the solution is based on the method of reversal, and the idea of the sequential access restriction is added. The transformation steps can be expressed as follows .

$\mathrm{L}$ is sequence of solutions, the partition results of cluster is known, and the new sequence of $\mathrm{L}$ after transformation can be solved by the following algorithm.

Step1. Divide the sequence (L) into K sub-sequences according to the number of nodes in each cluster, and orderly segmentation.

Step2. Generate two numbers randomly, respectively as the start and end position of the reverse operation.

Step3. Reverse the sequence between the above two numbers, and the reverse operation is indicated as follows:

Suppose there are 12 nodes assumed in the Euclidean plane, according to the convex hull can be divided into 3 clusters: $\{1,2,3,4,5\},\{6,7,8,9,10\}$ and $\{11,12\}$. The initial solution sequence consists of the 3 cluster nodes in an orderly combination, use $L$ to indicate it, $L=\{1,2,3,4,5|6,7,8,9,10|$ $11,12\}$, and "|" means the segmentation line of each cluster.

Now we will reverse the sequence from 3 to 9 . The reverse process is as follows:

(1) $\mathrm{L}=\{1,2,3,4,5|6,7,8,9,10| 11,12\}$ Segment the original solution according to the length of each cluster.

(2) $\mathrm{L}=\{1,2,3,4,5|6,7,8,9,10| 11,12\}$ Mark the location from 3 to 9 .

(3) $\mathrm{L}=\{1,2,5,4,3|9,8,7,6,10| 11,12\}$ Reverse the sequence within the same cluster.

After the operations, the new solution is $\{1,2,5,4,3,9,8,7,6,10,11,12\}$.

\section{Simulation Experiment}

Experimental Analysis. In order to test the feasibility and effectiveness of the proposed method for solving the TSP problem, We have chosen the international common TSP test database.

The simulation of the experimental environment is MATLAB, we have carried out the experiment on the data set of TSP Lib.

The experiment parameter Settings: the initial temperature $\mathrm{T} 0=1000$, the termination temperature Tend $=0.001$, cooling rate $\mathrm{Q}=0.9$, the Metropolis iterations $\mathrm{L}=200$. One of the optimal solutions 
of oliver30 stochastic time is as follows:

$26,20,18,15,12,14,9,8,5,4,2,6,7,10,3,1,13,11,17,16,19,24,25,29,28,21,22,23,27,30$.

The length of this path is $\mathrm{L}=423.7406$, and the optimal solution is obtained in the sixtieth iteration (as shown in Fig. 4).
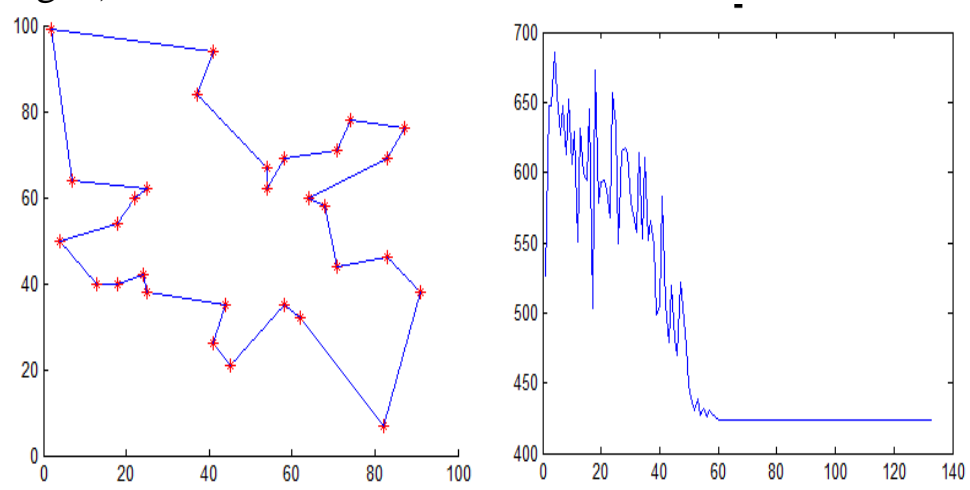

Figure 4. Final loop and iteration number of Olivr30

The final travel route and convergence algebra of rand50 are as follows (shown in Fig. 5).The final path length is 5555.5254, the actual optimal solution is 5553, and the error is very small.
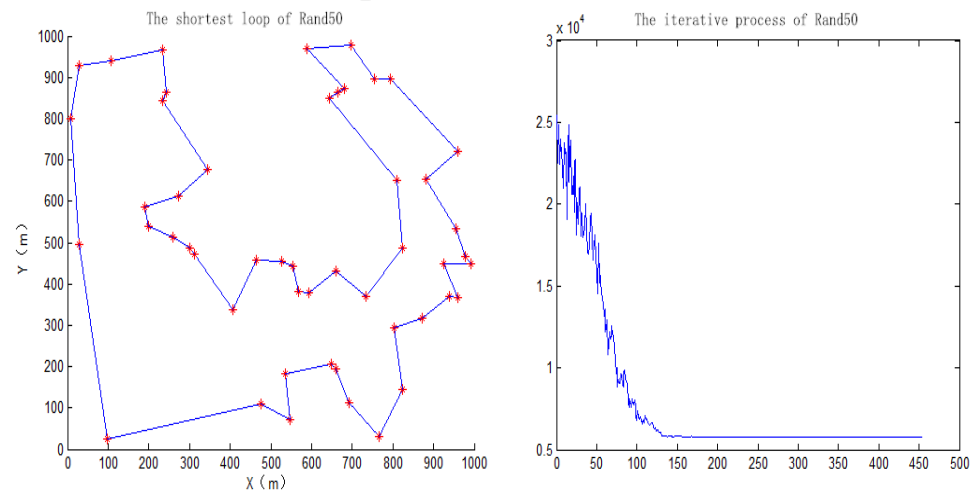

Figure 5. Final loop and iteration number of Rand50

We made 100 experiments with the above method, and compared with the general simulated annealing algorithm, genetic algorithm and ant colony algorithm.

At last, we make a statistics on the results of the above experiments (including the worst solution _worst, the optimal solution _best, the average solution Avg_l and the average number of iterations Avg_t), and the results of the experiments are as follows (as shown in Table 1).

Table 1 Comparison of oliver30 experimental data

\begin{tabular}{ccccc}
\hline Algorithm & _worst & _best & Avg_length & Avg_iterations \\
\hline GA & 489 & 422 & 456 & 1000 \\
ACA & 440 & 421 & 432 & 88 \\
SA & 439 & 420 & 427 & 100 \\
Algorithm in this paper & 429 & 420 & 425 & 70 \\
\hline
\end{tabular}

In order to better reflect the superiority of the improved SA algorithm, we carried out a series of experiments, and compared the experimental convergence of data set oliver30 in TSP Lib (as shown in Fig. 6).

As you can see from the figure, compared with the traditional SA algorithm, the improved SA algorithm is convergent in advance. The initial solution and the fluctuation range of the solution is small in improved SA algorithm, while the initial solution and the fluctuation range of the annealing process is quite large in traditional SA algorithm.

For typical examples of TSP Lib, we carried out experiments on a number of data sets in TSP 
Lib , respectively, with the traditional SA algorithm and the improved SA based on sequential access restrictions. The experimental results are as follows (as shown in Table 2). The best result _best, the worst result _worst, the average result Avg_length, the average iteration number Avg_iteration and the experimental deviation $\sigma \%$ in 30 experiments were recorded in this table.

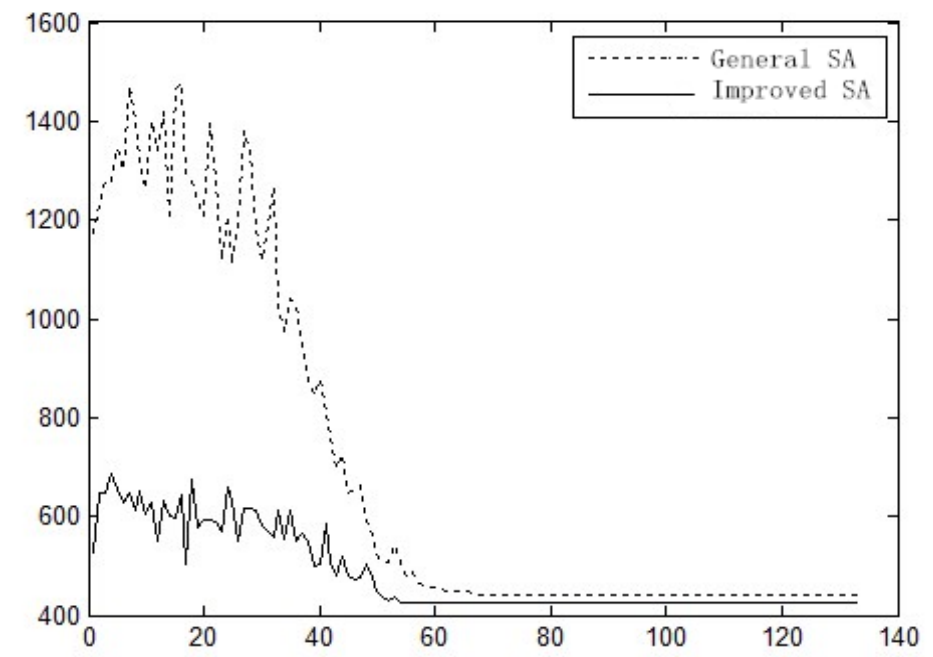

Figure 6. Comparison of general SA and improved SA

The experimental deviation computation formula is as follows:

$$
\sigma \%=\frac{\sum_{i=1}^{T}\left(S_{i}-S_{o p t}\right)}{T \times S_{o p t}} \times 100 \%
$$

Among them, $\mathrm{T}$ is the number of independent experiments, $\mathrm{Si}$ is the shortest path length of each experiment, Sopt is currently known as the shortest path length in TSP Lib.

Table 2 Comparison of experimental data

\begin{tabular}{|c|c|c|c|c|c|c|c|c|c|c|c|}
\hline \multirow{2}{*}{ Data Sets } & \multirow{2}{*}{$S_{\text {opt }}$} & \multicolumn{6}{|c|}{ general SA algorithm } & \multicolumn{4}{|c|}{ improved SA algorithm } \\
\hline & & best & _worst & Avg_1 & $\sigma \%$ & Avg_t & best & _Worst & Avg_1 & $\sigma \%$ & Avg_t \\
\hline Oliver30 & 420 & 420 & 439 & 427 & 1.67 & 88 & 420 & 429 & 425 & 1.19 & 70 \\
\hline Eil51 & 426 & 426 & 455 & 442 & 3.75 & 124 & 426 & 449 & 437 & 2.58 & 84 \\
\hline Rand50 & 5553 & 5767 & 6112 & 5970 & 7.51 & 178 & 5632 & 5860 & 5763 & 3.78 & 97 \\
\hline Rand75 & 7054 & 7223 & 7804 & 7552 & 7.06 & 193 & 7120 & 7628 & 7416 & 5.13 & 113 \\
\hline Rand100 & 7891 & 8369 & 8958 & 8641 & 9.50 & 275 & 8127 & 8573 & 8419 & 6.69 & 225 \\
\hline
\end{tabular}

The Superiority of the Algorithm. Through the comparison of the experimental data analysis, the proposed SA algorithm based on sequential access restrictions is better than the traditional SA algorithm, has faster convergence rate and faster search to the optimal solution in solving combinatorial optimization problems. And in the accuracy of the solution, able to obtain the better approximate optimal solution.

\section{Summary}

This paper put forward a new method of dividing clusters based on the sequential access restrictions theorem for TSP problem, applies the clustering results to the traditional simulated annealing algorithm, and improves the transformation method of the solution. In order to verify the effectiveness of this algorithm, we have compared the traditional SA algorithm and the improved SA algorithm, make statistics on the results of the experiments, and the experimental results show that the improved SA algorithm has better convergence speed and search accuracy. When the data is large and the nodes are centered on the convex hull, it cannot be simply divided into different 
clusters according to the convex hull vertex. In the future study, we will improve the algorithm by combining the density-based clustering method.

\section{Acknowledgements}

The National Natural Science Foundation of China (31201121).

Provincial Teaching Research Project of Hubei Province (2012215).

\section{References}

[1] E. L. Lawler, J. K. Lenstra, A. H. G. R. Kan, and D. B. Shmoys, The Traveling Salesman Problem: A Guided Tour of Combinatorial Optimization. Wiley, 1985.

[2] Steinbrunn M, Moerkotte G, Kemper A. Heuristic and Ran2 domized Optimization for the Join Ordering Problem [J]. The VLDB Journal, 1997, 6 (3):8 - 17.

[3] XU Xiao-Ping, ZHU Qiu-Qiu. Improved Simulated Annealing Algorithm of Solving TSP [J]. Computer Systems \& Applications, 2015, 12:152-156.

[4] Yang Wei-bo, ZHAO Yan-wei. Improved simulated annealing algorithm for TSP [J]. Computer Engineering and Applications, 2010, 15:34-36.

[5] LIU Yi,XIONG Sheng-wu. Hybrid tabu-simulated approach to solve traveling salesman problem [J]. Computer Engineering and Applications, 2009, 31:43-45.

[6] Mu hui, Yang Shao-wei. An Adaptive Simulated Annealing Genetic Hybrid Algorithm [A]. Intelligent Information Technology Application Association. Applied Computing, Computer Science, and Computer Engineering (ACC 2011 V4) [C].Intelligent Information Technology Application Association: 2011, 6.

[7] Lu Dan, Tong Chuang-ming, ZHONG Wei-jun. Study on hybrid algorithm based on particle swarm optimization and simulated annealing algorithm [J]. Computer Engineering and Design, 2011, 02:663-666.

[8] Liu Jin. Applied Research of Hybrid Genetic Algorithm and Simulated Annealing Algorithm in Traveling Salesman Problem [D]. South China University of Technology, 2014.

[9] LIU Zhen, CHEN Yan-hao, XIAO Wen-xian. One-way-ring shuffled frog leaping algorithm based on simulated annealing and its application to optimization problems [J]. Journal of Anhui University (Natural Science Edition), 2013, 05:25-31.

[10] Xu Sheng, Ma Xiaojun, Qian Hai, Wang Zhenyu. Genetic-simulated Annealing-based Ant Colony Algorithm for Traveling Salesman Problem [J]. Computer Measurement \& Control, 2016, 03:143-144+148.

[11]ZHOU Tao, LU Huiling. Clustering algorithm research advances on data mining. Computer Engineering and Applications, 2012, 12: 100-111.

[12]M. M. Flood, “The traveling salesman problem”, Operations Research, Vol. 4, pp. 61-75,1956. 